de la mutation p.y522S serait associée à une fuite de $\mathrm{Ca}^{2+}$ du RS responsable d'une activation de la nNOS (nitric oxide synthase) [9]. Les RNS produits entraîneraient une S-nitrosylation de la Cys 3635 de RYRl se traduisant par une augmentation de l'activation du canal muté à des températures supraphysiologiques. La fuite de $\mathrm{Ca}^{2+}$ du RS causée par la mutation p.y522S serait par ailleurs responsable d'un stress oxydatif chronique générateur d'altérations mitochondriales, elles-mêmes susceptibles d'amplifier le stress oxydatif et d'enclencher un cycle délétère stress-dommages (Figure 2). Il est cependant à noter qu'aucune altération significative des mitochondries n'a été observée dans les muscles des patients porteurs de la mutation p.y522S, ni décrite dans les muscles de nombreux autres patients porteurs de mutations de type HM.

\section{Conclusions et perspectives}

Des travaux récents réalisés chez la souris et chez l'homme montrent qu'en réponse à un exercice physique intense,
RYRl serait le siège d'un important remodelage comportant d'une part une phosphorylation de la Ser 2843 dépendante de la PKA (protein kinase A) et une S-nitrosylation des Cys 3635 et 2327 , et d'autre part une déplétion en FKBP12 [10]. La fuite importante du $\mathrm{Ca}^{2+} \mathrm{du}$ RS au travers de ces RYRl «remodelés» pourrait être la cause de dommages musculaires via une activation de la calpaïne par le $\mathrm{Ca}^{2+}$ et être l'un des composants de la limitation à l'exercice des muscles squelettiques.

Ces différentes données montrent que les modifications de l'état redox de RYRI jouent un rôle important dans la modulation de son activité canal. Les différents événements impliqués dans le remodelage de RYRl normal ou muté pourraient constituer des cibles thérapeutiques comme le suggère l'action du JTV519, une 1,4-benzothiazépine qui provoque la fixation de FKBP12 sur RYRl et qui améliorerait la fonction musculaire squelettique dans un modèle d'infarctus post-myocardique chez le rongeur [10]. $\diamond$

Ryanodine receptor:

redox state matters

\section{RÉFÉRENCES}

1. Lacampagne A, Fauconnier J, Richard S. Récepteur de la ryanodine et dysfocntionnement myocardique. Med Sci (Paris) $2007 ; 24: 399-405$.

2. Sun J, Xu L, Eu JP, et al. Classes of thiols that influence the activity of the skeletal muscle calcium release channel. J Biol Chem 2001 ; 276 : 15625-30.

3. Aghdasi, B, Reid MB, Hamilton SL. Nitric oxide protects the skeletal muscle $\mathrm{Ca}^{2+}$ release channel from oxidation induced activation. J Biol Chem $1997 ; 272: 25462-7$.

4. Eu JP, Sun J, Xu L, et al. The skeletal muscle calcium release channel: coupled $\mathrm{O}_{2}$ sensor and $\mathrm{NO}$ signaling functions. Cell $2000 ; 102: 499-509$.

5. Monnier N, Kozak-Ribbens G, Krivosic-Horber $R$, et al. Correlations between genotype and pharmacological, histological, functional and clinical phenotypes in malignant hyperthermia susceptibility. Hum Mutat $2005 ; 26$ : 413-25.

6. Chelu MG, Goonasekera SA, Durham WJ, et al. Heatand anesthesia-induced malignant hyperthermia in an RyRl knock-in mouse. FASEB J 2006 ; 20 : 329-30.

7. Quane KA, Keating KE Healy JMS, et al. Mutation screening of the RYRl gene in malignant hyperthermia: detection of a novel Tyr to Ser mutation in a pedigree with associated cores. Genomics $1994 ; 23$ : 236-9.

8. Monnier N, Krivosic-Horber R, Payen JF, et al. Presence of two different genetic traits in malignant hyperthermia families. Anesthesiology 2002 ; 97 : 1067-74.

9. DurhamWJ, Aracena-Parks P, Long C, et al. RyRl Snitrosylation underlies environmental heat stroke and sudden death in Y522S RyRl knockin mice. Cell 2008 ; $133:$ 53-65.

10. Bellinger AE, Reiken S, Dura M, et al. Remodeling of ryanodine receptor complex causes leaky channels: a molecular mechanism for decreased exercise capacity. Proc Natl Acad Sci USA 2008 ; 105 : 2198-2202.

\section{NOUVELLE}

\section{Inflammation intestinale} et stress du réticulum endoplasmique

\section{Un lien génétique}

Eric Chevet
Avenir Inserm U889, Université Bordeaux 2, 146, rue Léo Saignat, 33076 Bordeaux, France eric.chevet@u-bordeaux2.fr

Elle facilite le repliement, l'export et la dégradation des protéines dans des conditions de stress [3]. Dans les cellules de mammifères, la réponse UPR est sous le contrôle de trois protéines transmembranaires du $R \varepsilon$, dont l'inositol requiring enzyme-1 alpha and beta (IREl $\alpha \beta)$. IREl est la protéine initiatrice de la voie de signa- 
lisation de I'UPR la plus conservée au cours de l'évolution. Elle possède dans son domaine cytosolique une activité kinase et une activité endoribonucléase dont l'activation lors du stress du $R \varepsilon$ contribue à l'épissage non conventionnel d'un intron de 26 nucléotides dans I'ARNm codant pour la protéine $\chi$-box binding protein-1 (XBPl). Cela conduit à un changement du cadre de lecture et à la traduction d'une nouvelle protéine, dont les propriétés de facteur de transcription conduisent à l'induction d'un groupe de gènes cibles de l'UPR.

Jusqu'à aujourd'hui, I'UPR a été impliquée dans de nombreux phénomènes physiologiques et physiopathologiques incluant les maladies neurodégénératives, le diabète ou encore les cancers $[4,5]$. Dans un article récent, Arthur Kazer et al., démontrent pour la première fois le rôle clé joué par le stress $\mathrm{du} R \varepsilon$ dans les maladies inflammatoires de l'intestin [6]. En effet, des souris déficientes pour l'expression de XBP-1 dans les cellules épithéliales intestinales (CEl, dont il existe 4 types: cellules neuroendocrines, cellules à mucus, entérocytes, et cellules de Paneth) développent spontanément une entérite due à la baisse de la capacité des $C દ \mid$ à générer une activité antimicrobienne (cytokines) et à répondre de manière appropriée aux signaux inflammatoires locaux (protéines anti-inflammatoires). II est intéressant de recouper ces informations avec les données obtenues chez des souris déficientes pour l'expression de IREl $\beta$, normalement restreinte au tractus gastro-intestinal, qui développent une hypersensibilité à la colite induite par le sulfate de dextran [7]. Cette découverte a été complétée par l'identification de polymorphismes touchant un nucléotide unique (SNP) rares dans le gène codant pour XBPl et dont la présence confère un risque pour la maladie de Crohn (MC) et la colite ulcéreuse (CU) dans

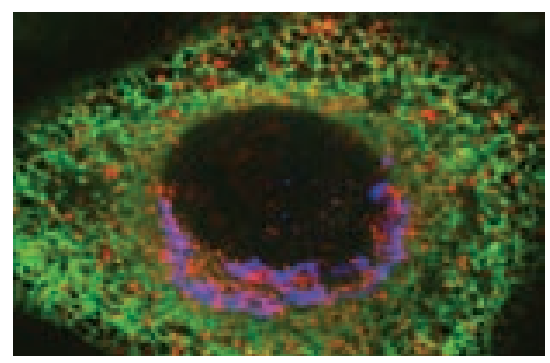

une cohorte de patients comprenant plus de 5000 contrôles et 4000 malades. Par conséquent, ce travail établit pour la première fois la contribution génétique du stress du RE aux maladies inflammatoires de l'intestin chez l'homme [6].

Ces résultats semblent donc indiquer une responsabilité des voies de stress du RE (et en particulier de l'axe IREl/ $X B P 1$ ) dans les mécanismes inflammatoires dans les CEl. Qui plus est, des mutations somatiques présentes dans le gène codant pour IREl $\alpha$ ont été associées à des processus de carcinogenèse [8]. Dans de telles conditions, pourquoi ne pas rechercher systématiquement d'éventuelles mutations dans les gènes codant pour les intermédiaires de signalisation de la voie IREl dans des maladies présentant des signes d'inflammation chronique associée à des cellules ayant une fonction sécrétrice essentielle? ou bien chercher des modulateurs pharmacologiques de cette voie de signalisation dont l'intérêt thérapeutique pourrait s'avérer réel ? $\diamond$ Intestinal inflammation and endoplasmic reticulum stress: a genetic link

\section{RÉFÉRENCES}

1. Todd DJ, Lee AH, Glimcher LH. The endoplasmic reticulum stress response in immunity and autoimmunity. Nat Rev Immunol 2008 ; 8 : 663-74.

2. Zhang K, Kaufman RJ. From endoplasmic-reticulum stress to the inflammatory response. Nature 2008; 454: 455-62.

3. Ron D, Walter P. Signal integration in the endoplasmic reticulum unfolded protein response. Nat Rev Mol Cell Biol 2007 ; 8 : 519-29.

4. Marciniak SJ, Ron D. Endoplasmic reticulum stress signaling in disease. Physiol Rev 2006; 86 : 1133-49.

5. Moenner M, Pluquet 0 , Bouchecareilh $M$, Chevet $\varepsilon$. Integrated endoplasmic reticulum stress responses in cancer. Cancer Res 2007 ; 67 : 10631-4.

6. Kaser A, Lee AH, Franke A, et al. XBPI links ER stress to intestinal inflammation and confers genetic risk for human inflammatory bowel disease. Cell 2008 ; $134: 743-56$.

7. Bertolotti A, Wang X, Novoa I, et al. Increased sensitivity to dextran sodium sulfate colitis in IRElbeta-deficient mice. J Clin Invest 2001 ; $107:$ 585-93.

8. Greenman C, Stephens P, Smith R, et al. Patterns of somatic mutation in human cancer genomes. Nature 2007 ; 446 : 153-8.

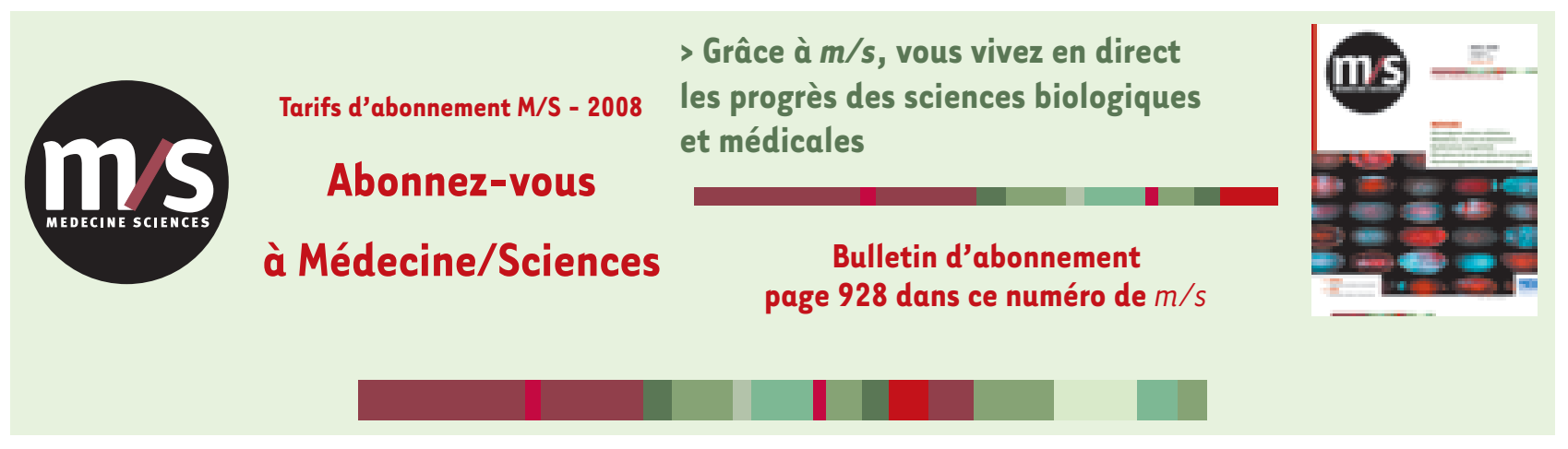

